cardiomyopathies are rather more superficial. The repeated reference in the former chapter to 'Berger' muscular dystrophy suggests little personal familiarity with genetic disorders.

Taken as a whole this is a book with a lot of valuable information in it, in keeping with the high standard always set by this series.

The publishers may wish to note two points. Firstly, the type setting is associated with a gross excess of hyphenation, which I have noticed in previous books produced by the same publisher. The use of automated typesetting techniques is no excuse for such an irritating fault. Secondly, the price is $£ 52$ which will severely limit the number of people purchasing this book and many libraries will decline to take it in these economically difficult times.

If the publishers really wish the book to sell they should have faith in their editors and contributors and produce it at a lower price for a wider audience. This series is too important to be relegated to obscurity by being priced out of the market.

Peter S Harper

\section{A Colour Atlas of Clinical Genetics}

By Michael Baraitser and Robin M Winter. (Pp 159; figures + tables. $£ 35$.) London: Wolfe Medical Publications. 1983.

This compact volume contains an abundance of excellent illustrations in a well designed format, placing emphasis on clinical photographs rather than descriptive text.

In the first five chapters the basic principles of genetics are described in a simple way for those not primarily involved in clinical genetics. Chapters 6 and 7 cover chromosome disorders and dysmorphic syndromes, while the remaining 12 chapters deal with disorders of particular systems, such as bone dysplasias, eye, skin, neurological, and muscle disorders.

Throughout the book the text highlights key features and the inheritance pattern of each disorder and many classification tables are included. A particularly useful practical guide to risk estimation and counselling in congenital deafness is given in chapter 9.

This book is a most helpful addition to current publications on syndrome identification, bringing together extensive experience of both rare and eommon disorders. It will be of value not only to geneticists and paediatricians, but also to obstetricians, general practitioners, students, and nurses. It is small enough to carry around easily and should be available in all out-patient clinics where these types of disorders are encountered, as well as paediatric and maternity wards, where it will provide a most useful source to aid diagnosis.

Helen M Kingston

\section{Developments in Human Reproduction and their Eugenic and Ethical Implications}

Edited by C O Carter. (Pp 256; figures + tables. $£ 18 \cdot 50, \$ 32 \cdot 00$.) London: Academic Press. 1983.

It is curious that, judging by exposure in the media, developments in human reproduction and the "new genetics' appear to generate more controversy and debate than issues such as global starvation and the prospect of nuclear annihilation, which would seem to be much more pressing. Yet it is a fact that much of society is more preoccupied with the possible problems of tomorrow than with those facing it today, so it is appropriate that these Proceedings of the 19th Annual Symposium of the Eugenics Society should be published in this well produced, relatively inexpensive, and highly readable volume.

The papers presented fall roughly into three groups. The first deals with the physiological and technological aspects of contraception, AID, in vitro fertilisation, and in vitro culture. All of these papers recognise the ethical difficulties and the Galton Lecture by Dr Edwards is particularly thoughtful. The second group relates to clinical genetics in its wider sense as it impinges upon the fetus, society, and the extended family. The concluding papers deal directly with the eugenic, ethical, and tangled legal implications of the new technology and its application.

This book is both instructive and stimulating and should do much to dispel the unfounded anxieties and misconceptions of those who fear that we are on the brink of a Brave New World. It can be readily recommended to all those concerned with the broader implications of medical genetics.

I D Young

\section{Hypermobility of Joints}

By P Beighton, R Grahame, and $\mathrm{H}$ Bird. (Pp xiii + 178; figures + tables. DM 110.) Berlin, Heidelberg, New York: Springer-Verlag. 1983.

This little book of 170 pages ranges widely over the whole field of diseases, disorders, and disasters that may lead to an unusual degree of joint mobility, from Charcot's joints through rheumatoid arthritis, traumatic rupture of ligaments, and various genetic disorders, to simple double jointedness. It is written 